\section{Revista 2020 \\ Med Vol. 28(2)}

julio-diciembre
- ISSN: 0121-5256 - e-ISSN: 1909-7700 =
Editorial Neogranadina

\title{
Anovulación y amenorrea secundaria: enfoque fácil y práctico*
}

\author{
Luis Ernesto Pérez Agudeloa
}

\begin{abstract}
Resumen: la anovulación ocurre en el $30 \%$ de la infertilidad y la amenorrea secundaria en el $4 \%$ de mujeres en edad reproductiva. Ambas alteraciones se pueden tornar crónicas conllevando riesgos de hiperestrogenismo e hipoestrogenismo crónicos. Tanto la anovulación como la amenorrea secundaria tienen las mismas causas endocrinas, excepto que la amenorrea tiene una adicional de causa uterina. No obstante existen muchas clasificaciones etiológicas, unas combinan la amenorrea secundaria con la primaria, otras se enfocan más a la anovulación y algunas agrupan las etiologías en compartimentos, sin lograr hacerlo en forma integral. El objetivo es revisar las clasificaciones etiológicas de anovulación y amenorrea secundaria, junto con la actualización de cada una de esas causas, a fin de seleccionar la clasificación que las agrupe integralmente para enfocar el manejo en forma fácil y práctica. Para ello, se revisó la literatura médica reciente en libros de texto, PubMed, Ovid, Clinical guidelines y Cochrane library, con palabras claves. Se concluye que la clasificación etiológica integral en cinco compartimentos es una guía fácil de seguir, para lograr el diagnóstico de cada una de esas causas, actualmente, cuando existen medicamentos y procedimientos quirúrgicos seguros para manejarlas como anovulación o como amenorrea secundaria.
\end{abstract}

Palabras clave: amenorrea, amenorrea secundaria, anovulación, anovulación crónica, clasificación de amenorrea

Recibido: 21/11/2019. Aceptado: 08/10/2020.

Disponible en línea: 04/06/2021

Cómo citar: Pérez Agudelo LE. Anovulación y amenorrea secundaria: enfoque fácil y práctico. Rev. Med. [Internet]. 4 de junio de 2021 [citado 4 de junio de 2021];28(2):85-102. Disponible en: https:// revistas.unimilitar.edu.co/index.php/rmed/article/view/4853

* Artículo de revisión.

a Profesor titular de Ginecología y Obstetricia, Universidad Militar Nueva Granada. Bogotá, Colombia. Correo electrónico: luis.perez@unimilitar.edu.co ORCID: https://orcid.org/0000-0002-1765-7645 


\title{
Anovulation and secondary amenorrhea: easy and practical approach
}

\begin{abstract}
: anovulation occurs in $30 \%$ of infertility and secondary amenorrhea in $4 \%$ of women in reproductive age. Both alterations can become chronic, carrying risks of chronic hyperestrogenism and hypoestrogenism. Both anovulation and secondary amenorrhea have the same endocrine causes, except that amenorrhea has an additional uterine cause. However, there are many etiological classifications, some combine secondary and primary amenorrhea, others focus more on anovulation and some group the etiologies into compartments, without achieving a comprehensive approach. The objective is to review the etiological classifications of anovulation and secondary amenorrhea, together with the update of each one of these causes, in order to select the classification that can integrally groups them so that to their management can be focused on an easy and practical way. For this purpose, recent medical literature in textbooks, PubMed, Ovid, Clinical guidelines and Cochrane library was reviewed with key words. It is concluded that the comprehensive etiological classification in five compartments is an easy guide to follow to achieve the diagnosis of each of these causes, currently, when there are drugs and safe surgical procedures to manage them as anovulation or secondary amenorrhea.
\end{abstract}

Keywords: amenorrhea, secondary amenorrhea, anovulation, chronic anovulation, amenorrhea classification

\section{Anovulação e amenorreia secundária: abordagem fácil e prática}

Resumo: a anovulação ocorre em 30 \% da infertilidade e a amenorreia secundária em 4 \% de muIheres em idade reprodutiva. Ambas as alterações podem se tornar crônicas, implicando riscos de hiperestrogenismo e hipoestrogenismo crônicos. Tanto a anovulação quanto a amenorreia secundária têm as mesmas causas endócrinas, exceto que esta última tem ainda causa uterina. Contudo, existem muitas classificações etiológicas, umas combinam a amenorreia secundária com a primária, outras se focam mais na anovulação, e algumas agrupam as etiologias em compartimentos sem poder fazer isso de forma integral. O objetivo é revisar as classificações etiológicas de anovulação e amenorreia secundária, junto com a atualização de cada uma dessas causas, a fim de selecionar a classificação que as agrupe integralmente para focar o manejo de forma fácil e prática. Para isso, foi revisada a literatura médica recente em textos consultados em PubMed, Ovid, Clinical guidelines e Cochrane library, a partir de palavras-chave. Conclui-se que a classificação etiológica integral em cinco compartimentos é um guia fácil de seguir para obter o diagnóstico de cada uma dessas causas, atualmente, quando existem medicamentos e procedimentos cirúrgicos seguros para manejá-las como anovulação ou como amenorreia secundária.

Palavras-chave: amenorreia, amenorreia secundária, anovulação, anovulação crônica, classificação de amenorreia 


\section{Introducción}

El ciclo menstrual es la manifestación periódica mensual que realiza el sistema reproductor femenino en favor de la iniciación de un embarazo. Los ciclos ovulatorios normales se caracterizan por su regularidad, con fases lúteas de catorce días y niveles de progesterona mesoluteínica entre 10 y 20 $\mathrm{ng} / \mathrm{mL}(1)$.

Anovulación es la falta de liberación de ovocitos por los ovarios. Es un desorden que ocurre en el 25-30\% de la infertilidad. La anovulación está presente en el $5 \%$ de mujeres con ciclos regulares, cuando cursan con fases lúteas inadecuadas y baja producción de progesterona, y en el 100\% de mujeres con ciclos frecuentes, infrecuentes y amenorreas. La anovulación se torna crónica cuando persiste por más de seis meses, conllevando alteraciones endocrinas con tres riesgos fundamentales: (i) hiperplasia endometrial secundaria a hiperestrogenismo crónico sin oposición con progesterona; (ii) igualmente, resistencia a la insulina y enfermedad cardiovascular, y (iii) osteoporosis, en caso de hipoestrogenismo crónico. Por otro lado, la infertilidad anovulatoria constituye un motivo de consulta en continuo crecimiento, debido a que las mujeres buscan embarazarse a edades superiores a los 35 años, cuando la reserva ovárica entra en disminución permanente, como se puede ir constatando con la paralela disminucón de folículos antrales y de la hormona antimülleriana (НАM) (2).

Amenorrea secundaria es la falta de menstruación por tres meses consecutivos, en mujeres con ciclos previos regulares, o de 6 meses con ciclos irregulares, aunque es más práctico considerar solo 3 meses en todos los casos. La amenorrea secundaria tiene una prevalencia del 3-4\%, ocasionada por las mismas causas endocrinas de la anovulación, más un adicional de alteraciones uterinas. Por lo tanto, la prevalencia de anovulación y amenorrea secundaria son diferentes; además, porque las pacientes consultan unas veces por infertilidad anovulatoria y otras veces por amenorrea.

\section{Metodología}

Este artículo es una revisión, con el objetivo de actualizar el enfoque y manejo de la anovulación y amenorrea secundaria. Para ello, se revisó la literatura médica de los últimos 10 años, en libros de texto, pubMed, Ovid, Clinical guidelines y Cochrane library, desde el primero de enero del 2010 hasta junio del 2020, en idiomas español e inglés, teniendo en cuenta las palabras clave. Finalmente se seleccionaron 3 libros de texto, de autores conocidos, con artículos pertinentes y 46 de 60 artículos (Figura 1).

Búsqueda de literatura en: pubMed, Ovid, Clinicas guides y Cochrane libary; con las palabras clave: amenorrea, amenorrea secundaria, anovulación, anovulación crónica, clasificación de amenorrea

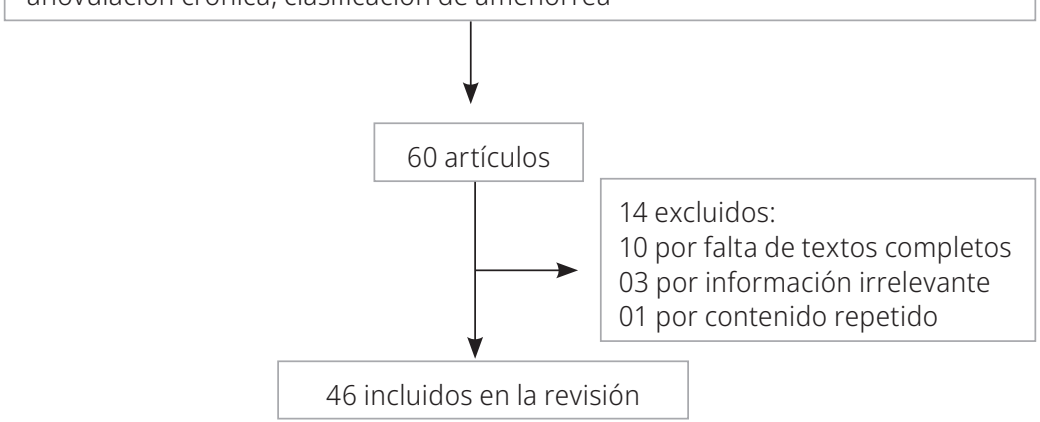

Figura 1. Flujograma de la literatura revisada. Fuente: elaboración propia.

Con los 5 artículos en libros de texto, y los 46 seleccionados en el flujograma, se obtuvo suficiente información para: (i) seleccionar la clasificación más didáctica, (ii) actualizar cada una de las causas, tanto de anovulación como de amenorrea secundaria y (iii) agrupar integralmente las causas, a fin de hacer un manejo de cada una de ellas con un enfoque comprensible, fácil y práctico. 


\section{Clasificación de la anovulación y amenorrea secundaria}

En primer lugar, la amenorrea es primaria o secundaria. Sin embargo, es más prudente separar sus manejos. Si bien es cierto que, en general, tiene causas similares, es más prudente y fácil clasificarlas y manejarlas en forma separada. A juicio de muchos, es mejor clasificar la amenorrea primaria dependiendo de la presencia o ausencia de senos y de útero, dado que esos fenotipos femeninos muchas veces tienen cariotipos masculinos, $46 \mathrm{XY}$ (3).

En segundo lugar, ante una paciente con amenorrea secundaria, lo primero que el médico debe hacer es descartar: (i) causas fisiológicas, como embarazo, lactancia y perimenopausia; (ii) causas iatrogénicas, como progestágenos, GnRHa, anticonceptivos hormonales, radioterapia, quimioterapia, etc., y (iii) amenorrea por enfermedades sistémicas, como alcoholismo, drogadicción, enfermedad hepática crónica, falla renal crónica, infecciones graves, inmunodeficiencias, etc.

Finalmente, nos quedamos con la anovulación y la amenorrea secundaria, ocasionadas por patologías endocrinas del eje hipotálamo-hipófisis-ovario (нно), de la tiroides y de la cápsula suprarrenal, y una adicional de amenorrea de causa uterina. Para ellas hay numerosas clasificaciones, entre las que sobresalen la de la Organización Mundial de la Salud (oms), la de la Sociedad Americana de Medicina Reproductiva y la clasificación en compartimentos.

\section{Clasificación de la oms}

Incluye 7 grupos de amenorrea. Se presentará entre paréntesis el porcentaje de anovulaciones correspondiente a cada grupo (4).

Grupo 1. Hipogonadotrópica: falla hipotálamo-hipofisario (10\%)

Grupo 2. Normogonadotrópica: disfunción hipotálamo-hipofisaria (85\%)

a) Disfunción central (10\%)

b) Síndrome de ovario poliquístico (70\%)

Grupo 3. Hipergonadotrópica: falla gonadal (4\%) Grupo 4. Alteraciones uterinas

Grupo 5. Prolactinoma

Grupo 6. Hiperprolactinemia funcional

Grupo 7. Tumores hipotálamo-hipofisarios no hiperprolactinémicos
Esta clasificación presenta la tendencia de agrupar la anovulación en tres grupos: grupo I (hipogonadotrópicas), grupo II (normogonadotrópicas), grupo III (hipogonadotrópicas). Pero no incluye hipotiroidismo, hiperplasia adrenal, Síndrome de Cushing, ni tumores productores de andrógenos. Además, pone al síndrome de ovario poliquístico (sop) como una disfunción hipotalámica, a pesar de ser una compleja endocrinopatía en la que el ovario ocupa un protagonismo hiperandrogénico (5).

\section{Clasificación de la Sociedad Americana de Medicina Reproductiva}

Incluye una amplia distribución topográfica de difícil seguimiento, por incluir en un mismo grupo patologías muy heterogéneas (6).

\section{Clasificación en compartimentos}

De Speroff: mezcla primaria con secundaria en cuatro compartimentos ascendentes desde el punto de vista anatómico: útero, ovario, hipófisis e hipotálamo, desconociendo las causas tiroideas y suprarrenales (2).

De la sociedad española de fertilidad: las ubica en cuatro compartimentos descendentes, pero sin incluir causas tiroideas ni suprarrenales (7).

Clasificación en 5 compartimentos: publicada en 1993 por Rebar, ya integrando clínicamente la tiroides y la suprarrenal (8). No obstante, en Colombia la hemos ido modificando y actualizando, acorde a los avances en laboratorio hormonal e imagenología, hasta agrupar todas las causas de anovulación y amenorrea secundaria en 5 compartimentos, incluyendo la causa uterina de amenorrea. Así, queda completa, sencilla, fácil y práctica (Tabla 1) (1, 9-12).

\section{Disfunción hipotalámica hipofisiaria (DHH)}

Es una alteración que inicialmente tiene anovulación en ciclos regulares con gonadotropinas y estrógenos en los límites bajos de normalidad, después pasa a ciclos infrecuentes y, según la severidad, llega a amenorrea hipogonadotrópica-hipoestrogénica. Causa el 10\% de la anovulación y el 15\% de la amenorrea. Se caracteriza por la triada de estrés, alteraciones de peso y ejercicio físico intenso (13). 
El estrés físico y mental, fundamentalmente, producen aumento de hormona liberadora de corticotropina (CRH, por sus siglas en inglés), activando la producción de proopiomelanocortina, de donde se derivan las $\beta$-endorfinas que inhiben cantidad y frecuencia en bolos de GnRH; también por estrés hay producción de otros neurotransmisores inhibidores, como dopamina y serotonina. Adicionalmente, la DHH en mujeres jóvenes puede asociarse a problemas psiquiátricos, como bulimia y anorexia nerviosa. En consecuencia, a medida que van aumentándose los inhibidores del eje hipotalámico-hipofisiario-ovárico (HHO), van emergiendo ciclos menstruales con fase lútea inadecuada, ciclos menstruales infrecuentes y anovulación con amenorrea hipogonadotrópica-hipoestrogénica, y cuando el hipoestrogenismo persiste por mucho tiempo, pueden aparecer disminución de la densidad mineral ósea y trastornos cardiovasculares, incluyendo anomalías endoteliales y cambios en el perfil lipídico (14). La DHH es responsable del 10$35 \%$ de la amenorrea secundaria, afectando aproximadamente a 1.6 millones de mujeres en EEUU y 17.4 millones universalmente $(15,16)$.

\section{Diagnóstico}

Síntomas y signos: según la severidad, pueden abarcar desde ciclos normales frecuentes o infrecuentes hasta amenorrea, en mujeres estresadas y con índice de masa corporal (IMC) alterado, incluyendo también obesas sin otra etiología endocrina.

Paraclínicos: FSH primero normal y después baja o normal, al igual que los estrógenos. La resonancia nuclear magnética (RNM) debe ser normal y necesaria para descartar LHH (Figura 2).

\section{Tratamiento}

El cambio de hábitos, hacia unos de vida saludable, puede ser la solución definitiva: dieta, ejercicio físico y manejo del estrés.

Anovulación: citrato de clomifeno o inhibidores de la aromatasa; o ampollas de FSH en pacientes hipogonadotropismo.

Amenorrea: se trata con anticonceptivos orales combinados (ACOS) o terapia hormonal cíclica de estrógenos-progestágenos (TEP) (Tabla 1)

Tabla 1. Anovulación y amenorrea secundaria, clasificación etiológica en cinco compartimentos, con sus respectivas prevalencias

\begin{tabular}{|c|c|c|c|}
\hline Compartimentos & Causas & Anovulación \% & Amenorrea \% \\
\hline \multirow{3}{*}{ Central } & $\mathrm{DHH}$ & 10 & 15 \\
\hline & $\mathrm{LHH}$ & 10 & 15 \\
\hline & Hiperprolactinemia & 17 & 20 \\
\hline \multirow{3}{*}{ Ovárico } & SOP & 70 & 25 \\
\hline & IOP & $10-30$ & 10 \\
\hline & Tumores Ags & - & 1 \\
\hline Tiroideo & Hipotiroidismo & 6 & 12 \\
\hline \multirow{3}{*}{ Suprarrenal } & Hiperplasia S & 1 & 0.5 \\
\hline & Tumores Ag & - & $<1$ \\
\hline & S. Cushing & - & 1 \\
\hline Uterino & & & 6 \\
\hline
\end{tabular}

Fuente: elaboración propia, a partir de (3-47). 


\section{Lesión hipotalámica hipofisiaria (LHH)}

Es una amenorrea hipogonadotrópica-hipoestrogénica causante del 10\% de la anovulación y del 15\% de la amenorrea. Múltiples lesiones estructurales del hipotálamo y la hipófisis son las responsables (17).

a. Adenomas: son el 91\% de masas selares y paraselares. Selares de TSH, de GH, de ACTH, prolactinomas, FSH-LH (raros).

b. Tumores: craneofaringioma, cordoma, metástasis, meningioma, tumor de células germinales, ependimoma, hemangioma, linfoma primario, sarcoma, carcinoma sinusal, papiloma, Schwannoma, pituicitoma.

c. Quistes: aracnoideo, de Rathke, dermoide, epidermoide, quiste supraselar, mucocele.

d. Lesiones vasculares: aneurisma, fístula arteriovenosa de senos cavernosos.

e. Procesos infiltrativos: sarcoidosis, histiositosis, displasia fibrosa, hemocromatosis, hiperplasia hipofisiaria, absceso, hipofisitis, sinusitis esfenoidal, tuberculosis, cisticercosis.

f. Otros: síndrome de silla turca vacía (S. Sheehan), radioterapia, isquemia e infarto, hipofisectomía.

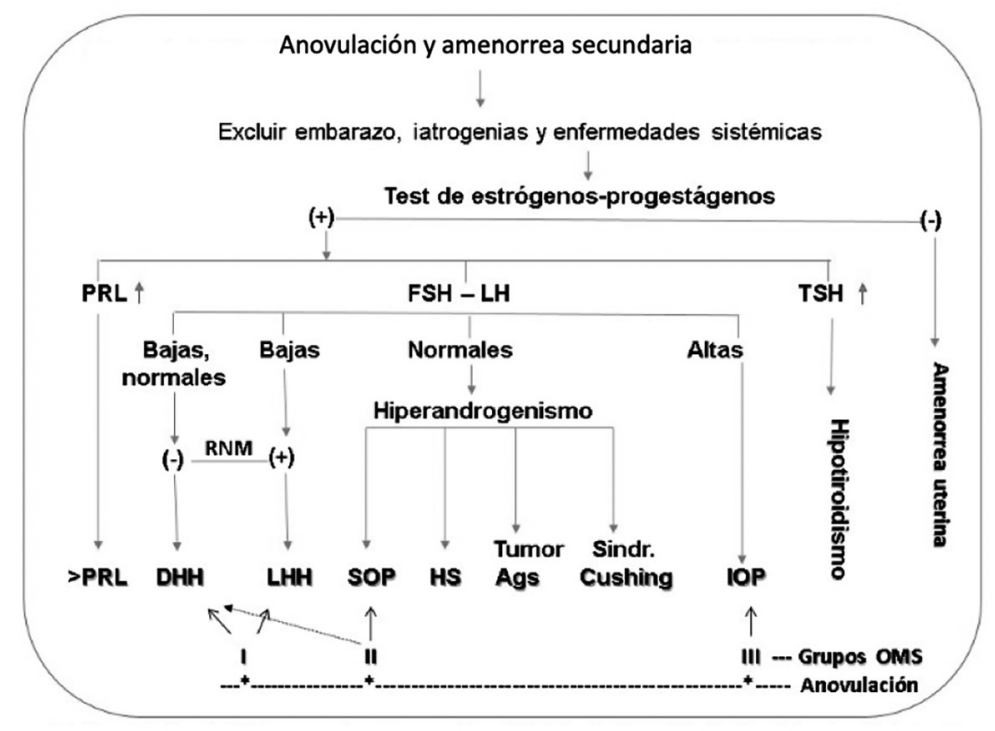

Figura 2. Enfoque de anovulación y amenorrea secundaria. > PRL hiperprolactinemia; RNM, resonancia nuclear magnética; $\mathrm{DHH}$, disfunción hipotálamo hipofisiaria; LHH, lesión HH; SOP, síndrome de ovario poliquístico; HS, hiperplasia suprarrenal; IOP, insuficiencia ovárica prematura. Fuente: elaboración propia, a partir de (12).

\section{Diagnóstico}

Signos y sintomas. Se presenta amenorrea acompañada de síntomas y signos neurológicos producidos por las lesiones estructurales $\mathrm{HH}$, tales como: cefalea, hipertensión endocraneana y alteraciones de pares craneanos, entre otras. En la evaluación, generalmente, es necesario el concurso de endocrinólogos, neurólogos, ginecólogos y neurocirujanos.
Paraclínicos:

- Gonadotropinas y estrógenos: bajos.

- RNM: muestra las masas expansivas y procesos infiltrativos.

- Test de GNRH : para diferenciar lesiones hipotalámicas de lesiones hipofisarias (bolo subcutáneo de 100 mg de GNRH y se determina la respuesta de LH y FSH basal, a los 30 ' y a los 60 '. Si se doblan los niveles de gonadotrofinas, 
tras el estímulo, se confirma integridad hipofisaria).

- Megatest de reserva hipofisaria: para determinar si hay otras líneas hormonales comprometidas (se administra TRH + GNRH + CRH + GHRH y se valora la respuesta de TSH, LH, FSH, PRL, cortisol y GH).

\section{Tratamiento}

El tratamiento de la lesión generalmente es quirúrgico. Después de la cirugía, muchas pacientes quedan con panhipopituitarismo y necesidad de terapia de reemplazo hormonal para los ejes comprometidos: levotiroxina, para el tiroideo; corticoides, para el suprarrenal, y TEP para el ovárico.

Anovulación: etiológico e inducción de ovulación con gonadotropinas.

Amenorrea: etiológico y TeP (Tabla 2).

Tabla 2. Tratamiento de la anovulación y de la amenorrea secundaria.

\begin{tabular}{|c|c|c|c|}
\hline & \multicolumn{2}{|c|}{ Anovulación } & \multirow{2}{*}{ Amenorrea } \\
\hline & Primera línea & Segunda línea & \\
\hline DHH & Estilo de vida saludable, Letrazol, CC & FSH amp & Estilo de vida saludable, TEP, ACOS \\
\hline LHH & Etiológico, FSH/LH amp & - & Etiológico,TEP \\
\hline$>$ PRL & Etiológico, Agonistas DA, Letrazol/CC & FSH/LH amp & Etiológico, TEP, ACOS \\
\hline SOP & Letrazol, Letrazol/CC & FSH amp, Drilling & ACOS \\
\hline IOP & FIV-Ovocitos donados & FIV-Ovocitos estimulados & TEP \\
\hline T. Androgénico & Cirugía, inducción? & - & Cirugía, TEP? \\
\hline Hipotiroidismo & Levotiroxina & Letrazol, CC & Levotiroxina \\
\hline HS & Dexametasona & Letrazol, CC & Dexametasona, ACOS \\
\hline S. Cushing & Cirugía, Inhibidores esteroides & Inducción acorde & Cirugía, ACOS \\
\hline Uterina & & & Cirugía, trasplante? \\
\hline
\end{tabular}

LHH, lesión HH; DHH, difusión HH;>PRI, hiperprolactinemia; DA, dopamina; IOP, insuficiencia ovárica prematura TEP, terapia estrógenosprogestágenos; CC, citrato de Clomifeno. ACOS, Anticonceptivos orales combinados. Fuente: elaborado a partir de (12-48)

\section{Hiperprolactinemia (>PRL)}

La hiperprolactinemia es producida por numerosas causas, periféricas y centrales. No obstante, los prolactinomas hipofisarios constituyen más de la tercera parte de las amenorreas centrales, por lo cual se colocan en este compartimento, en donde hay otras lesiones tumorales, que por efecto de masa las producen. Las causas periféricas son fisiológicas, iatrogenias y enfermedades crónicas (Tabla 3). El principal control fisiológico endocrino de la PRL es ejercido por la dopamina (DA) producida en el mesencéfalo. La DA llega al núcleo arcuato e inhibe la producción de $\mathrm{GnRH}$, y pasa por la eminencia media al sistema porta hasta llegar a inhibir los receptores D2 del lactotropo hipofisario. Otros controladores de menor importancia son el tono opioide, disminución del GAP, y la presión de pseudoprolactinomas sobre los axones dopaminérgicos a nivel tubero-infundibular. La inhibición del eje Hнo se va acentuando de acuerdo con los niveles de PRL, resultando con ello, desde ciclos menstruales regulares con fase lútea inadecuada y ciclos infrecuentes, hasta amenorrea.

La PRL es una molécula monomérica de 23 $\mathrm{kDa}$. Sin embargo, pueden estar circulando en sangre moléculas grandes de $50 \mathrm{kDa}$ y grandes-grandes de más de $100 \mathrm{kDa}$ constituyendo 
la macroprolactinemia. Estas macromoléculas producen confusión, porque son competentes inmunológicamente, dejándose medir, pero biológicamente incompetentes y por lo tanto no producen síntomas; sin embargo, pueden estar asociadas a la PRL Anormal, produciendo síntomas con niveles muy altos de PRL, por lo cual se debe descartar la macroprolactinemia en el laboratorio (18).

\section{Diagnóstico}

Signos y sintomas: ciclos infrecuentes, amenorrea, galactorrea (56\%) más asociada a hiperprolactinemia tumoral en los que además pueden encontrase efectos de masa.

\section{Paraclínicos:}

- Prolactina: normal (pool). Cuando está entre 20 y $50 \mathrm{ng} / \mathrm{mL}$ se trata de hipotiroidismo, iatrogenia y trastornos funcionales; entre 50 y $100 \mathrm{ng} / \mathrm{mL}$ pueden ser microprolactinomas; $y>100 \mathrm{ng} / \mathrm{mL}$ son microprolactinomas, macroprolactinomas, o pseudoprolactinomas. En un estudio multicéntrico de 233 adolescentes, se encontraron niveles promedios de PRL de $69 \mathrm{ng} / \mathrm{mL}$ en trastornos iatrogénicos, $93.15 \mathrm{ng} / \mathrm{mL}$ en microprolactinomas y $241 \mathrm{ng} / \mathrm{mL}$ en macroprolactinomas (19).
- Macroprolactina: se puede medir en el laboratorio, cuando la PRL sea mayor de $100 \mathrm{ng} / \mathrm{mL}$ en pacientes asintomáticas y sintomáticas con posible asociación.

- RNM: diagnostica prolactinomas (40\% de adenomas hipofisarios) y pseudoprolactinomas.

- Campimetría o potenciales visuales evocados: pueden mostrar alteraciones visuales en caso de macroprolactinomas o pseudoprolactinomas.

- Megatest de reserva hipofisiaria: ocasionalmente, es prudente realizarlos.

\section{Tratamiento}

Debes ser etiológico, con agonistas dopaminérgicos o cirugía. La bromocriptina y la cabergolina son los agonistas más usados. Se administran teniendo en cuenta tolerancia, riesgos y disponibilidad. Se inician a bajas dosis (1,25 cada noche de bromocriptina o $0.25 \mathrm{mg}$ dos veces por semana de cabergolina) y se van aumentado hasta obtener la respuesta deseada. La bromocriptina también se puede aplicar intravaginalmente. Pero, usualmente, se prefiere cabergolina, por causar menores reacciones colaterales gastrointestinales y requerir solo dos dosis orales por semana, aunque se debe considerar el riesgo rarísimo de hipertrofia valvular cardiaca.

Tabla 3. Causas de la hiperprolactinemia

\begin{tabular}{|l|l|}
\hline Fisiológicas & $\begin{array}{l}\text { Embarazo (excepcional en amenorrea primaria) } \\
\text { Ejercicio } \\
\text { Coito } \\
\text { Estimulación de las mamas (succión) } \\
\text { Estrés }\end{array}$ \\
\hline Macroprolactinemia(10\%) & IgG - Ligada a la PRL \\
\hline Lesiones hipotalámico- & $\begin{array}{l}\text { Prolactinomas (micros o macros) } \\
\text { Masas no funcionales: Adenoma, craniopharyngioma, meningioma, quistes. } \\
\text { Lesiones inflamatorias/infiltrativas: Hipoficitis linfocitica, histiocitosis. }\end{array}$ \\
\hline Otras enfermedades & Hipotiroidismo, falla renal crónica. \\
\hline Medicamentos & $\begin{array}{l}\text { Antisicóticos (risperidone, amisulpride, paliperidone, haloperidol) } \\
\text { Antieméticos (metoclopramide, domperidone) } \\
\text { Antidepresivos } \\
\text { Opioides } \\
\text { Estrogenos } \\
\text { Antihipertensivos (verapamil) }\end{array}$ \\
\hline
\end{tabular}




\section{Hiperprolactinemia fisiológica: retirar}

la causa (prótesis mamaria, cicatrices en la línea mamaria, estímulo del pezón, etc.). Coadyuvar, si es necesario, con agonistas dopaminérgicos o clomifeno para la anovulación, y con Acos para la amenorrea.

\section{Hipotiroidismo: levotiroxina.}

\section{Prolactinomas:}

- Cabergolina, como primera línea. La respuesta es favorable en el $100 \%$ de microprolactinomas y $70 \%$ de macroprolactinomas. Se van aumentando las dosis, controladas con PRL semanalmente para ajustarlas; y si responden, se disminuye la PRL, la paciente menstrua entre 3 y 10 semanas y los macroadenomas disminuyen de tamaño al control de RNM o TAC a las 6 semanas. Siguiendo controles RNM a los 3 meses y anualmente, se puede bajar dosis después de un año y retirar, o descansar, si a los dos años la RNM es normal.

- Resección tras esfenoidal, si no toleran agonistas dopaminérgicos, para 30\% de macroprolactinomas que no responden a los agonistas, y para los pseudoadenomas. Pero, puede haber resecciones incompletas, recidivas y panhipopituitarismo posterior.

- Radioterapia, como tercera línea de manejo, para recidivas.

- Amenorrea: posterior a las resecciones de prolactinomas, en general las pacientes quedan con hipopituitarismo, en terapia hormonal de reemplazo con TEP, levotiroxina y corticoides.

- Anovulación: Letrazole, clomifeno o gonadotropinas (en caso de hipopituitarismo).

Prolactinomas y embarazo: los microprolactinomas crecen 3-5\% durante el embarazo, por lo que se suspenden los agonistas dopaminérgicos; pero, los macroprolactinomas crecen un 15-20\% durante el embarazo y sí ameritan continuar el tratamiento.

\section{Síndrome de ovario poliquístico (SOP)}

Es la anormalidad endocrina más común de la mujer, tanto en anovulación como en amenorrea (Tabla 1). Se encuentra en el $10-15 \%$ de mujeres en edad reproductiva, dependiendo de la población estudiada. Se asocia a amenorreas secundarias $(25 \%)$, anovulación crónica $(70 \%)$, obesidad (40-80\%), hirsutismo (50-80\%), ciclos infrecuentes (80-90\%) y resistencia a la insulina (52\%). Genera alto riesgo futuro de síndrome metabólico, incluyendo DM2, hipertensión y ateroesclerosis (20).

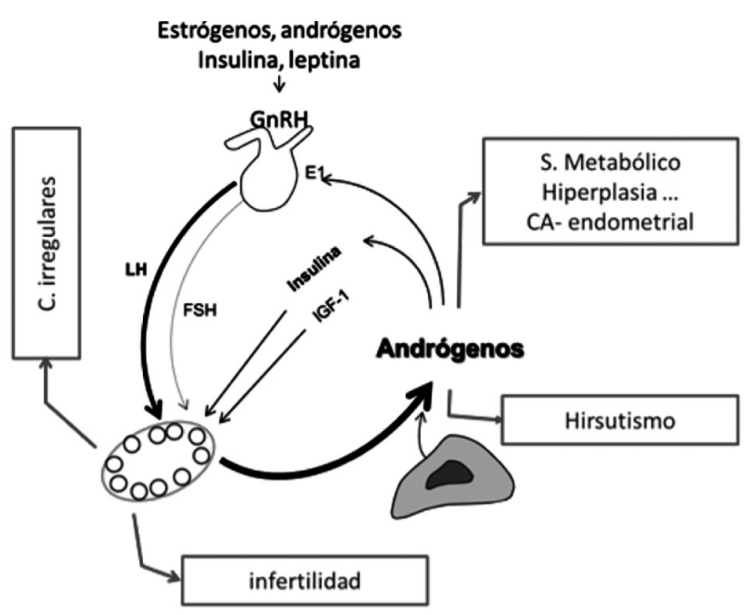

Figura 3. Fisiopatología del Síndrome de Ovario Poliquístico. Fuente: elaboración propia.

Fisiopatología: en el sop se encuentran varias alteraciones derivadas de factores genéticos y ambientales (Figura 3) (12, 21-23):

- Mayor producción de LH que de FSH.

- Aumento en la frecuencia de producción de GNRH, secundaria a la disminución de dopamina $\mathrm{y}$ al aumento de leptina e insulina.

- Hiperinsulinemia y aumento de la IGF-1, debido a factores genéticos y ambientales (dieta y ejercicio) que resultan en: (i) aumento de ácidos grasos libres; (ii) alteración de la fosforilación de los sustratos de receptores de insulina, con serina a cambio de tirosina y la consiguiente alteración 
del glut-4 para internalizar la glucosa en las células, y (iii) presencia de marcadores de inflamación crónicos: CPR, ferritina, transferrina, leucocitos, TNFa, IL6, IL18, PAI1 y haptoglobina en cadena $\alpha$.

- Aumento en la producción de andrógenos ováricos, por estimulación de la teca externa con LH e insulina, y dificultad de su aromatización en la granulosa ante las bajas concentraciones de FSH e interferencia de IGF-1 en sus receptores (24).

- Anovulación crónica al detenerse la maduración folicular en estadio antral, con acumulación de quistes foliculares menores de $10 \mathrm{~mm}$.

- Manifestación clínica de infertilidad, hirsutismo, ciclos infrecuentes y amenorrea.

- Riesgos de diabetes II, síndrome metabólico, enfermedad cardiovascular, acantosis nigricans, cambios de humor, apnea obstructiva del sueño, hiperplasia endometrial y carcinoma de endometrio.

Tabla 4. Criterios diagnósticos del soP

\begin{tabular}{l|l}
\hline Grupo de trabajo & \multicolumn{1}{c}{ Criterios } \\
\hline N/H & $\begin{array}{l}\text { 1. Hiperandrogenismo clínico y } \\
\text { bioquímico } \\
\text { 2- Anovulación crónica (ciclos } \\
\text { irregulares) }\end{array}$ \\
\hline $\begin{array}{l}\text { Dos de los primeros tres criterios: } \\
\text { 1. Hiperandrogenismoclínico y } \\
\text { bioquímico }\end{array}$ \\
$\begin{array}{l}\text { 2. Anovulación crónica (ciclos } \\
\text { Societies }\end{array}$ & $\begin{array}{l}\text { irregulares) } \\
\text { Roterdam 2003arios poliquísticos } \\
\text { 4. Excluir otras causas de anovulación } \\
\text { con hiperandrogenismo }\end{array}$ \\
\hline $\begin{array}{l}\text { AES \& PCOS } \\
\text { Societies 2009 }\end{array}$ & $\begin{array}{l}\text { 1. Hiperandrogenismo clínico y } \\
\text { bioquímico } \\
\text { 2. Anovulación crónica (ciclos } \\
\text { irregulares) y/o ovarios poliquísticos } \\
\text { 3. Excluir otras causas de anovulación } \\
\text { con hiperandrogenismo }\end{array}$ \\
\hline $\begin{array}{l}\text { NIH: National Institute of Health; ESHRE: European Society of } \\
\text { Human Reproduction and Embriology; ASRM: American Society } \\
\text { of Reproductive Medicine; AES and PCOS: Androgen Excess and } \\
\text { Polycistic Ovarlan Syndrome. Fuente: elaborado a partir de (12). }\end{array}$ \\
\hline
\end{tabular}

\section{Diagnóstico}

Es fácil cuando se tienen en cuenta los criterios diagnósticos (Tabla 4). Sin embargo, aún se discute si son más apropiados, en el diagnóstico de sop, los criterios de Rotterdam o los de Tesaloniqui. En los criterios de Rotterdam del 2003, además de considerar los fenotipos que origina, se considera el diagnóstico con 2 de los 3 criterios iniciales, más el cuarto criterio. Los tres criterios de Tesaloniqui, realizados por la sociedad de exceso de andrógenos y la sociedad de sop en 2009, al parecer son los más simples y apropiados (25):

1. Evidencia clínica o bioquímica de exceso de andrógenos.

2. Trastornos del ciclo y/o, ovarios poliquísticos (más de 12 micro quistes subcapsulares menores de $10 \mathrm{~mm}$ y volumen ovárico mayor de $10 \mathrm{cc}$ ).

3. Exclusión de otras causas de anovulación, relacionadas con exceso de andrógenos: hiperplasia suprarrenal, tumor productor de andrógenos, síndrome de Cushing, hiperprolactinemia e hipotiroidismo.

La resistencia a la insulina se presenta en el 51\% de los casos. Es evidente cuando hay acantosis nigricans o cuando el test Homa (del inglés, homeostatic model assessment) es mayor a 3 (insulinita basal x glicemia basal en mg/dL / $405=<3$ es lo normal). Sin embargo, no es un criterio diagnóstico de sop (25).

\section{Tratamiento del sop}

Manejo básico para todas las pacientes: (i) estilo de vida saludable, que incluye dieta y ejercicio físico para mantener un IMC normal. Cirugía bariátrica si IMC $>35 \mathrm{~kg} / \mathrm{m}^{2}$. Con solo reducir 5\% se aumenta la frecuencia de la ovulación (26). (ii) Metformina: para pacientes que tengan resistencia a la insulina, generalmente obesas, con hiperandrogenismo bien manifiesto y antecedentes familiares. Se benefician, especialmente, disminuyendo los riesgos de DM2 y de síndrome metabólico (27).

\section{Tratamiento de la anovulación}

En el 2010, la medicina basada en evidencias había colocado al citrato de clomifeno como la primera línea de tratamiento de la infertilidad, con iguales tasas de embarazos frente al letrozol (inhibidor de la aromatasa), pero menos costoso que este. Sin 
embargo, en 2017 un metanálisis concluyó que el clomifeno no es tan bueno, superándolo la combinación de clomifeno-metformina o de solo Letrazole (28). Y los metanálisis 2018 y 2019 del grupo Cochrane, con 42 RCT en 7935 pacientes, concluyeron que el letrozol es mejor que el clomifeno en tasas de embarazos (OR 1.56, 95\% CI 1.37 - 1.78) y en tasas de recién nacidos vivos (OR 1.68, 95\% CI 1.42 - 1.99) $(29,30)$.

Por consiguiente, en la actualidad se maneja la anovulación, así (31-33):

a. Primera línea, Letrazole.

b. Segunda línea, Letrazole-clomifeno o solo clomifeno.

c. Tercera línea, ampollas de FSH o drilling ovárico por laparoscopia.

\section{Tratamiento de la amenorrea}

ACOs con progestinas antiandrogénicas. Útiles para manejar el hirsutismo al que se le agrega manejo cosmético facial con Eflornithine en crema y ocasionalmente espironolactona oral.

\section{Insuficiencia ovárica prematura (IOP)}

La insuficiencia ovárica prematura (IOP) o insuficiencia ovárica primaria, antes llamada falla ovárica prematura (FOP), es la pérdida temporal o definitiva de la función ovárica, en menores de 40 años. Esta amenorrea hipergonadotrópica-hipoestrogénica (Figura 2). Es potencialmente reversible, dado que $5-10 \%$ vuelven a tener ciclos menstruales intermitentes y embarazos espontáneos en 4.4\%, razón por la cual la denominación IOP es más apropiada (34, 35). La prevalencia de IOP en general es del $1 \%$ en sobrevivientes de cáncer; pero, en general es del $3.7 \%$ y relacionada con la edad: entre 18 y 25 años $1 / 10.000$, entre 25 y 30 años $1 / 1.000$, y entre 35 y 40 años $1 / 100$. Ocasiona por lo menos el $10 \%$ de anovulación y de amenorrea. Muchas IOP eran consideradas idiopáticas o iatrogénicas; sin embargo, los adelantos en secuenciación genética y biología molecular han ido aumentado sus causas, así: a. Iatrogénicas (50\%): oforectomías, radioterapia, quimioterapia, terapia uterina embolizante, endometriosis.

b. Autoinmunes (15-30\%): acompañando a síndromes poliendocrinos autoinmunes (tipos I y II), S. de Smith, insuficiencia suprarrenal primaria, hipotiroidismo, etc.

c. Genéticas (30\%): síndrome del X frágil, síndrome de Turner, síndromes genéticos (ataxia telangiectásica, síndrome de Fanconi, envejecimiento prematuro), entre otras

d. Metabólicas: galactosemia

е. Infecciosas: parotiditis, HIV, твC, malaria, herpes, citomegalovirus

f. Tóxicas y ambientales: pesticidas, anestésicos, tabaco

\section{Diagnóstico}

Síntomas y signos: el hipoestrogenismo agudo ocasiona síntomas vasomotores y, si es crónico, pone a todas las pacientes en riesgo de vaginitis atrófica, trastornos de la libido, alteraciones psicológicas, osteoporosis y enfermedad cardiovascular.

Paraclínicos: FSH elevado y estrógenos bajos (Tabla 5).

\section{Tratamiento}

Infertilidad: profilácticamente, con (i) criopreservación de embriones en pacientes con posibilidad de compañero masculino y (ii) criopreservación de ovocitos o de tejido cortical ovárico, en pacientes jóvenes con sospecha familiar de IOP o esperando manejos oncológicos. Embarazo lo antes posible con: (i) transferencia de embriones o fertilización in vitro (FIV) con los embriones u ovocitos preservados; (ii) si no se hizo profilaxis, es mejor realizar lo más pronto posible FIV con ovocitos donados; no es prudente esperar reversión espontanea de la IOP, o estimulación de inductores de ovulación. (iii) Últimamente se está intentando, en pacientes con HAM positiva, extracción por laparoscopia de tejido cortical ovárico para fragmentación capsular, estimulación y autotransplante, 
esperando reversión de la IOP y hasta embarazos espontáneos o con fIV $(35,36)$.

Amenorrea: se trata con TEP cíclica.

\section{Tumores productores de andrógenos}

Los tumores que producen andrógenos excesivamente son ováricos o suprarrenales. Ocasionan desfeminización aguda, seguida de virilización y amenorrea. Representan el 1-2\% de la amenorrea con hirsutismo severo. Los tumores virilizantes ováricos pueden ser de Sertoli-Leydig, de la teca granulosa, de células lipídicas, de células hiliares, de restos adrenales, disgerminomas y gonadoblastomas $(37,38)$. Por su parte, los suprarrenales son adenomas y carcinomas (39).

\section{Diagnóstico}

Desfeminización, seguida de masculinización y amenorrea. Andrógenos muy altos. La ecografía muestra los tumores ováricos y la RNM los suprarrenales. Ocasionalmente, cuando la imagenología es negativa, se recurre al test de supresión ovárica con GnRHa para poder saber si son ováricos o suprarrenales.

\section{Tratamiento}

La resección quirúrgica resuelve la anovulación y la amenorrea. Los andrógenos bajan a niveles normales y el hirsutismo se resuelve paralelamente, cosméticamente.

\section{Hipotiroidismo}

El hipotiroidismo primario es el más frecuente (95\%) y en mujeres mucho más que en hombres, en proporción 10/1. Ocurre en el 6\% de la anovulación y hasta en el $12 \%$ de la amenorrea $(40,41)$. El hipotiroidismo secundario (hipofisario) y el terciario (hipotalámico) son raros.

El hipotiroidismo afecta al eje HHO de varias formas, hasta producir amenorrea:

a. Dopamina aumentada: la hormona tiroidea disminuida produce retroalimentación negativa, aumentando la TRH, con estímulo paralelo de TSH y
PRL, y la hiperprolactinemia aumenta la dopamina.

b. Esteroides sexuales aumentados: el hipotiroidismo produce disminución de la globulina trasportadora de hormonas sexuales (sHBG), con la consiguiente elevación de estrógenos y andrógenos libres. Igualmente, la aromatización periférica de andrógenos contribuye al hiperestrogenismo.

c. Pico ovulatorio de LH alterado que conducen a falta de estímulo sobre el cuerpo lúteo.

d. Autoinmunidad afectando tanto a la tiroides, como al ovario.

\section{Diagnóstico}

Las pacientes presentan amenorrea, obesidad, intolerancia al frío, retardo mental, ptosis palpebral, bradicardia, constipación, mixedema y piel seca. TSH > $10 \mathrm{mU} / \mathrm{mL}$ (entre 4 y 10 es subclínico), T4 libre baja, los anticuerpos antitiroideos (antitiroglobulina y antiperoxidasa) pueden estar positivos y la PRL elevada. El hipotiroidismo subclínico puede producir anovulación y el clínico anovulación y amenorrea.

\section{Tratamiento}

Levotiroxina hasta estabilizar la TSH $<2.5 \mathrm{mUI} /$ mL (Tabla 2). (i) Anovulación, ocasionalmente es necesario agregar letrazol o clomifeno. Amenorrea: si no revierte con levotiroxina, agregar Acos.

\section{Hiperplasia suprarrenal de aparición tardía}

La hiperplasia suprarrenal, productora de amenorrea secundaria, es secundaria a bloqueos parciales enzimáticos en la esteroidogénesis de las cápsulas suprarrenales. El bloque más frecuente es el de la 21-hidroxilasa, seguido por el de la 11 -hidroxilasa. Con estos bloqueos se disminuye la síntesis de cortisol, se produce retroalimentación negativa con aumento crónico de АCTH, hasta producir hiperplasia de las cápsulas suprarrenales. Esos bloques enzimáticos desvían el metabolismo hacia la producción de progesterona, 17 hidroxiprogesterona (17-OHP) y andrógenos, con cuadro clínico de hiperandrogenismo y anovulación crónica $(42,43)$. 


\section{Diagnóstico}

Los bloqueos severos se manifiestan en la recién nacida con deficiencias graves de cortisol y aldosterona que, al no ser tratadas, ponen en peligro la vida. Cuando se manifiesta en forma tardía, en la mujer adulta, lo hace con un cuadro parecido al del sop, con hirsutismo, ciclos infrecuentes y amenorrea. Pero se diferencia porque las pacientes tienen 17-OHP con niveles mayores a $10 \mathrm{ng} / \mathrm{mL}$ (normal $<2 \mathrm{ng} / \mathrm{mL}$ ). En casos de 17-оHр entre 2 y 10 (zona gris) se realiza estímulo con АCTH para desenmascarar el diagnóstico.

\section{Tratamiento}

Corticoides: dexametasona $0,5 \mathrm{mg} /$ día o su equivalente con prednisona (5 mg) o hidrocortisona (10 $\mathrm{mg}$ ). (i) En anovulación, ocasionalmente, es necesario agregar letrazol o clomifeno. (ii) La amenorrea se maneja con ACOS (43).

\section{Síndrome de Cushing}

El síndrome se caracteriza por síntomas y signos ocasionados por el exceso de cortisol en sangre. El hipercortisolismo se corrobora midiéndolo en orina de 24 horas o en saliva nocturna ( $11 \mathrm{pm})$. Tiene cuatro causas (44):

a. Iatrogénica, la más común

b. Adenomas suprarrenales: la АСтн es menor de 5 ng/mL y el TAC suprarrenal positivo para tumor

c. Tumor hipofisiario productor de АСТH (enfermedad de Cushing): la АСтн es mayor de $20 \mathrm{ng} / \mathrm{mL}$ y la RNM de hipófisis es positiva para tumor; en casos dudosos se miden gradientes de АСТH, cateterizando los senos venosos petrosos (45)

d. Producción ectópica de ACTH O CRH (tumor bronco génico): la RNM hipofisaria es normal y la АСтH alta no se deja inhibir con dosis elevadas de dexametasona

\section{Diagnóstico}

Es una amenorrea con hiperandrogenismo, como el sop, la hiperplasia y los tumores productores de andrógenos. Pero se diferencia de ellos porque las pacientes presentan fatiga persistente, depresión o cambios de humor, debilidad muscular, acumulación excesiva de grasa en mejillas (cara de luna llena) nuca (cuello de búfalo) y abdomen, estrías cutáneas, hirsutismo, presión arterial alta e hiperglicemia. Una vez establecido el hipercortisolismo, su diagnóstico diferencial se realiza con anamnesis, ACTH plasmática, prueba de $\mathrm{CRH}$, prueba de inhibición con dosis altas de dexametasona e imagenología. Ameritan remisión a Endocrinología, Medicina Interna o Cirugía.

\section{Tratamiento}

Etiológico, generalmente quirúrgico: sin embargo, inicialmente o cuando la cirugía no es exitosa, se trata con inhibidores de la síntesis esteroidea o antagonistas de los receptores de glucocorticoides (osilodrostat y levoketoconazole); además, cuando se extrae el adenoma hipofisario (enfermedad de Cushing), algunas mujeres quedan con hipopituitarismo, ameritando manejo de suplencias hormonales para ejes ovárico, tiroideo y suprarrenal.

Amenorrea: TEP

Anovulación: inducción con gonadotropinas (46).

\section{Amenorrea uterina}

La Amenorrea uterina sucede por falta de útero funcional, estando las pacientes endocrinológicamente normales. Ocurre por varias causas, en las cuales el test de estrógenos-progestágenos es negativo.

\section{Adherencias intrauterinas (síndrome de} Asherman)

Las adherencias obstruyen completamente la cavidad uterina, generalmente, secundarias a legrados enérgicos y repetidos en pacientes con abortos especialmente sépticos o por hemorragias puerperales. El síndrome de Asherman, ocurrido después de cesáreas o miomectomías, produce menstruaciones escasas, pero no amenorrea. El diagnóstico se hace por histerosonografia e histeroscopia. Y el tratamiento con adhesiolisis histeroscópica, complementado con balón de sonda de Foley intracavitaria, antibióticos y un ciclo de TEP con dosis altas de estrógenos (47). 


\section{Pérdida irreversible de la funcionalidad uterina}

- Destrucción endometrial secundaria a твC, radiación, ablación y terapia embolizante de leiomiomas.

- Necrosis uterina posterapia embolizante (48).

- Histerectomía subtotal desconocida por la paciente. Actualmente, es posible el trasplante uterino, para mujeres infértiles (49).

Necesitan útero subrogado o trasplante, en caso de infertilidad.

\section{Estenosis cervical}

Es una complicación rara de la conización cervical. Se produce amenorrea obstructiva con hematómetra dolorosa progresiva. La eco-tv hace el diagnostico. Se trata con dilatación cervical guiada por ecografía, sonda por una o dos semanas, antibióticos y un ciclo de TEP.

\section{Enfoque de la anovulación y amenorrea secundaria}

\section{Primera consulta}

Historia clínica: se descartan causas fisiológicas (no olvidar descartar embarazo), iatrogénicas y por enfermedades sistémicas crónicas. Se indaga por antecedentes que revelen anormalidades del eje endocrino reproductivo, tales como:

a. desarrollo de los caracteres sexuales secundarios

b. menarquia y características de los ciclos menstruales. Antecedentes gineco-obstétricos (intervenciones quirúrgicas, partos, hemorragia posparto, abortos, legrados)

c. historia sexual y métodos anticonceptivos

d. hirsutismo, acné, seborrea

e. galactorrea

f. enfermedades crónicas (diabetes mellitus, tiroiditis, enfermedad intestinal inflamatoria crónica, hepatopatías)

g. historia de traumatismos craneoencefálicos (infecciones, tumores del sistema nervioso central, galactorrea) h. tratamientos con quimioterapia y/o radioterapia

i. estilo de vida, ejercicio físico y hábitos alimentarios. Pérdidas importantes o fluctuaciones de peso. Estrés emocional.

En el examen físico, se deben relevar aspectos importantes:

a. índice de masa corporal (IMC) (peso / talla ${ }^{2}$ : normal 20 a $25 \mathrm{Kg} / \mathrm{m}^{2}$ )

b. perímetro abdominal (normal $<88 \mathrm{~cm}$ )

c. índice cintura/cadera (normal $<0.8$ )

d. hirsutismo, hipertricosis, alopecia, acantosis nigricans, acné, seborrea

e. campimetría por confrontación y valoración de movimientos oculares

f. tamaño y desarrollo de los senos (Tanner)

g. galactorrea

h. presencia de útero, a la especuloscopia y tacto bimanual

Perfil hormonal: muchas veces es posible, en la primera consulta, hacer un diagnóstico diferencial de la amenorrea. Sin embargo, se debe corroborar con perfil endocrino y test de útero funcional. Se solicitan las siguientes hormonas:

a. FSH, LH, PRL, TSHyE2 (si no hayhiperandrogenismo)

b. T total, T libre, DHEas y 17 oHp, en caso de haber encontrado signos o síntomas de hiperandrogenismo (acné, hirsutismo, virilización)

c. HAM (hormona antimülleriana) cuando la consulta es por anovulación y la paciente tiene más de 35 años de edad

Formulación del test de función uterina: en esa primera consulta, también, se formula un ciclo de estrógenos-progestágenos (Estradiol $2 \mathrm{mg}$ /día por 2 semanas + didrogesterona $10 \mathrm{mg} /$ día la segunda semana) para evaluar la función uterina (hacerlo con solo progestágeno puede dar falso negativo muchas veces). El test tiene dos efectos: (i) evalúa la existencia, o no, de útero funcional, y (ii) la mayoría de las veces, al tratarse de úteros funcionales, tranquiliza la paciente una vez que menstrúe. 


\section{Segunda consulta}

Diez días después de terminar el ciclo de estrógenos-progestágenos, si la paciente menstrúa por deprivación hormonal, se sabrá que el útero es normal. Si no sangra por deprivación, el diagnóstico es amenorrea uterina. Además, se evalúan los resultados del perfil hormonal (Tabla 5) y se confirma o cambia el diagnóstico realizado en la primera consulta. Se sigue el algoritmo de la Figura 2. Con este diagnóstico, en la mayoría de las veces, es posible iniciar el manejo correcto, bien sea de la amenorrea, bien de la infertilidad anovulatoria. No obstante, frecuentemente, es necesario profundizar la investigación en busca de una precisión diagnóstica, para poder dar el mejor manejo posible, con:

a. imagenología: (i) RNM ante la impresión diagnóstica de LHH, DHH, hiperprolactinemia, síndrome de Cushing, tumor androgénico suprarrenal; (ii) ecografía transvaginal, si sop.

b. otras hormonas o megatest endocrinos: en la mayoría de las veces es necesario corroborar las alteraciones hormonales encontradas; realizar megatest de hormonas liberadoras, para investigar compromisos de otras líneas hormonales; cortisol en orina de $24 \mathrm{hrs}$ o en saliva nocturna, si se piensa en S. de Cushing.

c. interconsultas: muchas veces, en casos de causas centrales y suprarrenales, se hará necesaria la interconsulta con psiquiatras, neurólogos, internistas, endocrinólogos y neurocirujanos.

\section{Tercera consulta}

Con la investigación realizada, ya se tendrá claro el manejo del diagnóstico etiológico, bien sea por anovulación, bien por amenorrea. Así, cubriremos todas las causas de anovulación y amenorrea secundaria.
Tabla 5. Valores hormonales

\begin{tabular}{|l|l|l|}
\hline Hormona & V. normal & Día del ciclo \\
\hline FSH & $3-10 \mathrm{mUI} / \mathrm{mL}$ & $3-5$ \\
\hline LH & $2-10 \mathrm{mUI} / \mathrm{mL}$ & $3-5$ \\
\hline PRL & $0-20 \mathrm{ng} / \mathrm{mL}$ & $3-5$ \\
\hline TSH & $0.2-3.7 \mathrm{mUI} / \mathrm{mL}$ & $3-5$ \\
\hline Estradiol & $25-75 \mathrm{pg} / \mathrm{mL}$ & $3-5$ \\
\hline Progesterona & $10-20 \mathrm{ng} / \mathrm{mL}$ & 21 (0 M-7) \\
\hline $17-0 H P$ & $<2 \mathrm{ng} / \mathrm{mL}$ & $3-5$ \\
\hline Testosterona libre & $0.2-2 \mathrm{pg} / \mathrm{mL}$ & $3-5$ \\
\hline Testosterona total & $15-70 \mathrm{ng} / \mathrm{dL}$ & $3-5$ \\
\hline DHEAS (20-30 años) & $65-270 \mu \mathrm{g} / \mathrm{dL}$ & $3-5$ \\
\hline DHEAS (30-49 años) & $45-240 \mu \mathrm{dL}$ & $3-5$ \\
\hline HAM & $0.7-3.5 \mathrm{ng} / \mathrm{mL}$ & Cualquiera \\
\hline Cortisol & $4-40 \mu \mathrm{g}$ & Orina/24 hrs \\
\hline
\end{tabular}

Fuente: elaborado a partir de $(50,51)$.

\section{Conclusión}

- La anovulación ocurre en el 30\% de parejas infértiles y la amenorrea secundaria en el $4 \%$ de mujeres en edad reproductiva.

- Si bien ambas pueden ser producidas por las mismas causas, la amenorrea presenta una causa uterina adicional en el $6 \%$ de las veces.

- La clasificación etiológica en cinco compartimentos es una guía fácil de seguir, para lograr un correcto diagnóstico diferencial y manejos apropiados. Mucho más si contamos con laboratorio de hormonas e imagenología.

- La amenorrea necesita tratamiento, y la anovulación, si hay infertilidad, para evitar los riesgos del hiperestrogenismo o del hipoestrogenismo crónicos. 
- La anovulación, muchas veces, puede ser manejada por ginecología general; pero la inducción monitorizada debe ser realizada por especialistas en infertilidad y endocrinología reproductiva.

\section{Referencias}

(1) Pérez LE, editor. Infertilidad y Endocrinología Reproductiva. Cuarta edición. Bogotá (Colombia): Brand Studio; 2015. Regulación neuroendocrinopsicoinmunológica del ciclo menstrual. p. 15-32.

(2) Taylor H S, Pal L, Seli E. Amenorrhea. En: Speroff's L, editor. Clinical Ginecology Endodocrinology and Infertility. $9^{a}$ ed. Philadelfia-Baltimor, New York, London, Buenos Aires, Hong Kong, Sydney, Tokio: Wolters Cluwer; 2020. p. 821-939.

(3) Jaimes H, Sepúlveda J. Amenorrea primaria. En: Pérez LE, editor. Infertilidad y Endocrinología Reproductiva. Cuarta edición. Bogotá (Colombia): Brand Studio; 2015. p. 153-167.

(4) Eshre Capri Workshop Group. Health and fertility in World Health Organization group 2 anovulatory women. Hum Reprod Update [Internet]. 2012;18(5):58699. doi: 10.1093/humupd/dms019.

(5) Loret de Mola R. Amenorrhea [Internet] 27 ago. 2016. Disponible en: https://abdominalkey.com/amenorrhea/\#bib3

(6) The Practice Committee of the American Society for Reproductive Medicine. Current evaluation of amenorrhea. Fertil Steril 2008; 90(suppl3):219-25.

(7) Protoloco sego. Amenorrea primaria y secundaria [Internet]. 2013;56(7):387-392. Disponible en: https:// www.elsevier.es/es-revista-progresos-obstetricia-ginecologia-151-articulo-protoloco-sego-amenorrea-primaria-secundaria--S0304501313000988.

(8) Rebar RW. Assessment of the female patient. En: Carr BR, Blackwell RE, editores. Textbook of reproductive Medicine. Norwalk UsA: Appleton \& Lange; 1993. p. 247-63.

(9) Pérez LE. Amenorrea secundaria. En: Fecolsog, editor. Texto de Obstetricia y Ginecología. 2a ed. Bogotá (Colombia): Alta voz; 2010. p. 727-732.

(10) Pérez LE., editor. Infertilidad y Endocrinología Reproductiva. $2^{a}$ ed. Bogotá (Colombia): Hospital Militar Central; 2000. Anovulación, oligomenorreas, amenorreas y hemorragia uterina disfuncional. p. 128-143.

(11) Pérez LE, editor. Infertilidad y Endocrinología Reproductiva. $3^{\mathrm{a}}$ ed. Barranquilla (Colombia): Grafimpresos; 2007. Anovulación y amenorrea secundaria. 169-184.

(12) Pérez LE, editor. Infertilidad y endocrinología reproductiva. $4^{\mathrm{a}}$ ed. Bogotá (Colombia): Universidad Militar Nueva Granada, Brand studio; 2015. Amenorrea secundaria y anovulación. 169-184.

(13) Mancini F, Martínez M, Tur R. Guía 6. Estudio y tratamiento de las amenorreas hipotalamohipofisarias. Hospital Universitario Quirón Dexeus [Internet]. Disponible en: https://www.sefertilidad.net/docs/biblioteca/guiasPracticaClinicas/guia6.pdf

(14) Vale B, Brito S, Paulos L, Moleiro P. Menstruation disorders in adolescents with eating disorders - target body mass index percentiles for their resolution. Einstein (São Paulo) [Internet]. 2017;12(2):175-180. Disponible en: https://www.ncbi.nlm.nih.gov/pubmed/25003922.

(15) Ackerman KE, Misra M. Amenorrhea in adolescent female athletes. Lancet Child Adolescent Health. 2018;2(9):677-688. doi: 10.1016/S2352-4642(18)30145-7.

(16) Kigawa M, Harada M, Kawahara T, Osuga Y, Saito S. Low bone mineral density in elite female athletes with a history of secondary amenorrhea in their teens. Clin J Sport Med. Clin J Sport Med. May. 2020;30(3):245250. doi:10.1097/JSM.0000000000000571.

(17) Shufelt CL, Torbati T, Dutra E. Hypothalamic amenorrhea and the long-term health consequences. Semin Reprod Med. 2017;35(3):256-262. doi: 10.1055/ s-0037-1603581.

(18) Vilar L, Vilar CF, Lyra R, Freitas MDC. Pitfalls in the diagnostic evaluation of hyperprolactinemia. Neuroendocrinol. 2019;109(1):7-19. doi: $10.1159 / 000499694$.

(19) Eren E, Törel Ergür A, İşgüven ŞP, Çelebi Bitkin E, Berberoğlu M, Şıklar Z, Baş F et al. Laboratory Characteristics of Hyperprolactinemia. DJ Clin Res [Internet]. 2019 may. 28;11(2):149-156. Disponible en: https://www.ncbi.nlm.nih.gov/pubmed/30396878.

(20) Escobar-Morreale HF. Polycystic ovary syndrome: definition, aetiology, diagnosis and treatment. Nat Rev Endocrinol. 2018; 14(5):270-284. doi: 10.1038/ nrendo. 
(21) Castro E. Síndrome de ovario poliquístico. En: Pérez LE, editor. Infertilidad y endocrinología reproductiva. $4^{a}$ ed. Bogotá (Colombia): Universidad Militar Nueva Granada, Brand studio; 2015. Pp. 217-243.

(22) Pasquali R, Gambineri A. New perspectives on the definition and management of polycystic ovary syndrome. J Endocrinol Invest. 2018; 41(10):1123-1135. doi: 10.1007/s40618-018-0832-1.

(23) Raperport C, Homburg R. The Source of Polycystic Ovarian Syndrome. Clin Med Insights Reprod Health. 2019;13:1179-1198. doi: 10.1177/1179558119871467.

(24) Rosenfield RL, Ehrmann DA. The pathogenesis of polycystic ovary syndrome (PCOs): The hypothesis of PCOS as functional ovarian hyperandrogenism revisited. Endocr Rev. 2016 oct.;7(5):467-520.

(25) Jeanes YM, Reeves S. Metabolic consequences of obesity and insulin resistance in polycystic ovary syndrome: diagnostic and methodological challenges. Nutr Res Rev. 2017;30(1):97-105.

(26) Jacob S, Balen A. How will the new global polycystic ovary syndrome guideline change our clinical practice? Clin Med Insights Reprod Health. 2019 jun.;13:17. doi: $10.1177 / 1179558119849605$.

(27) Morley LC, Tang T, Yasmin E, Norman RJ, Balen AH. Insulin-sensitising drugs (metformin, rosiglitazone, pioglitazone, D-chiro-inositol) for women with polycystic ovary syndrome, oligo amenorrhoea and subfertility. Cochrane Database Syst Rev. 2017;11:CD003053. doi: 10.1002/14651858. CD003053.pub6.

(28) Kim CH, Chon SJ, Lee SH. Effects of lifestyle modification in polycystic ovary syndrome compared to metformin only or metformin addition: a systematic review and meta-analysis. Sci Rep. 2020;10(1):7802. doi: 10.1038/s41598-020-64776-w.

(29) Wang R, Kim BV, van Wely M, Johnson NP, Costello $\mathrm{MF}$, Zhang $\mathrm{H}$ et al. Treatment strategies for women with WHO group II anovulation: systematic review and network meta-analysis. BMJ. 2017 en. 31;356:1-11. doi: 10.1136/bmj.j138.

(30) Franik S, Eltrop SM, Kremer JA, Kiesel L, Farquhar C. Aromatase inhibitors (letrozole) for subfertile women with polycystic ovary syndrome. Cochrane Database Syst Rev. 2018 may. 24; 5:CD010287. doi: 10.1002/14651858.CD010287.pub3.

(31) Mejia RB, Summers KM, Kresowik JD, Van Voorhis BJ. A randomized controlled trial of combination letrozole and clomiphene citrate or letrozole alone for ovulation induction in women with polycystic ovary syndrome. Fertil Steril. 2019 mar.;111(3):571-578. doi: 10.1016/j.fertnstert.2018.11.030.

(32) Costello MF, Misso ML, Balen A, Boyle J, Devoto L, Garad RM et al. A brief update on the evidence supporting the treatment of infertility in polycystic ovary syndrome. Aust N Z J Obstet Gynaecol. 2019 sep. 12;59(6). doi: 10.1111/ajo.13051.

(33) Teede H, Misso M, Costello $\mathrm{M}$ et al. Recommendations from the international evidence-based guideline for the assessment and management of polycystic ovary syndrome. Fertil Steril. 2018 ag.;110(3):364379. doi: 10.1016/j.fertnstert.2018.05.004

(34) Barreda-Velit C1, Salcedo-Pereda R, Ticona J. Schmidt's syndrome: a difficult diagnosis in the Latin American context. Rev Med Inst Mex Seguro Soc. 2018; 56(2):189-193.

(35) South Med J. 2017 Mar; 110 (3):147-153. Primary Ovarian Insufficiency: Current Concepts. Collins G1, Patel B1, Thakore S1, Liu J. doi: 10.14423/ SMJ.0000000000000611.

(36) Zhang X, Han T, Yan L, Jiao X, Qin Y, Chen ZJ. Resumption of ovarian function after ovarian biopsy/ scratch in patients with premature ovarian insufficiency. Reprod Sci. 2019 feb.;26(2):207-213. doi: $10.1177 / 1933719118818906$

(37) Cho K, Havelock JC, Gilks B, Dunne C. Case report: an identical twin with Sertoli-Leydig cell tumor. Gynecol Endocrinol. 2018 en. 24;34(7):563-566. doi: 10.1080/09513590.2018.1431215.

(38) Sehemby M, Bansal B, Sarathi V, Kolhe A, Kothari $\mathrm{K}$, Jadhav-Ramteke $\mathrm{S}$ el al. Virilising ovarian tumors: a single-center experience. Endocr Connect. 2018;7(12):1362-1369.

(39) LaVoie M, Constantinides V, Robin N, Kyriacou A. Florid hyperandrogenism due to a benign adrenocortical adenoma. вјм Case Rep. 2018 jul. 30;bcr2018224804. doi: 10.1136/bcr-2018-224804

(40) Poppe K. Velkeniers B. Thyroid disorders in infertile women. Ann Endocrinol. 2003;64(1):45-50

(41) Gronier H, Sonigo C, Jacquesson L. Impact of thyroid function on fertility. Gynecol Obstet Fertil. 2015 mar.;43(3):225-33. doi: 10.1016/j.gyobfe.2015.01.005

(42) Burdea L, Mendez MD. 21 Hydroxylase Deficiency. StatPearls [Internet] 2020 mar. 24 [revisión 2020 abr. 20]. Disponible en: https://www.ncbi.nlm.nih.gov/ pubmed/29630216 
(43) Pérez LE, editor. Infertilidad y endocrinología reproductiva. $4^{a}$ ed. Bogotá (Colombia): Universidad Militar Nueva Granada, Brand studio; 2015. Hiperplasia suprarrenal; 267-279.

(44) Barnett R. Cushing's syndrome. Lancet [Internet]. 2016 jul. 31 [revision 2020 may. 17];388(10045):649. Disponible en https://europepmc.org/article/ med/27551694. Doi: 10.1016/s0140-6736(16)31280-6.

(45) Feelders RA, Newell-Price J, Pivonello R, Nieman LK, Hofland LJ, Lacroix A. Advances in the medical treatment of Cushing's syndrome. Lancet Diabetes Endocrinol. 2019 abr.;7(4):300-312. doi: 10.1016/ S2213-8587(18)30155-4.

(46) Klibansky, A. Pregnancy after cure of Cushing's disease. Cushin's Support \& Research Foundation CSRF [Internet]. 2006 dic. 12. Disponible en: https://csrf. net/doctors-articles/reproductive-issues/pregnancy-after-cure-of-cushings-disease/

(47) Baradwan S, Baradwan A, Al-Jaroudi D. The association between menstrual cycle pattern and hysteroscopic march classification with endometrial thickness among infertile women with Asherman syndrome. Medicine (Baltimore). 2018;97(27):e11314. doi: 10.1097/MD.0000000000011314

(48) Mutiso SK, Oindi FM, Hacking N, Obura T. Uterine Necrosis after Uterine Artery Embolization for Symptomatic Fibroids. Case Rep Obstet Gynecol. 2018; 2018:9621741. doi: 10.1155/2018/9621741.

(48) Nobuhiko S, Ayako Hayashi, Iori Kisu, Kouji Banno, Hisako Hara, Makoto Mihara. Uterus transplantation: Toward clinical application in Japan. Reprod Med Biol. 2017;16(4): 305-313. doi: 10.1002/ rmb2.12048

(50) Salgado S. Análisis de hormonas femeninas en el estudio de fertilidad. Ovodonante [Internet]. 2016 nov. 21. Disponible en: https://ovodonante.com/analisis-de-hormonas-femeninas-en-el-estudio-de-fertilidad/

(51) Medilineplus.gov Medida de cortisol en orina. Mediplus Medical encyclopedia. 6 may. 2019 [Internet]. Disponible en: https://medlineplus.gov/spanish/ ency/article/003703.htm 